\title{
Nuestra opinión sobre la revisión sistemática "Glaucoma y antioxidantes: revisión sistemática" publicada en su revista
}

\section{Our opinion about systematic review "Glaucoma y antioxidantes: revisión sistemática" published in this journal}

\author{
Ilia A. Volchegorskii y Elena V.Tur* \\ South-Ural State Medical University, Chelyabinsk, Russia
}

Estimado Dr. Manuel Alejandro Garza León,

Hemos leído con gran interés la revisión sistemática de Giaquinta-Aranda, et al. ${ }^{1}$ publicada en las páginas de la revista que ustedes dirigen. Podemos decir que nos ha impresionado y causado gran respeto el planteamiento analítico de este trabajo y de su metodología.

Desafortunadamente, tras una lectura detallada del texto completo publicado, hemos encontrado contradicciones significativas y errores que, de hecho, podrían llevar al lector a malinterpretaciones y a dañar la reputación de la revista que publica esta revisión sistematizada.

Al comienzo de la sección de metodología de la revisión, aparece claramente indicado que, de acuerdo a los criterios de inclusión, "... publicaciones completas de ensayos clínicos controlados y aleatorizados..." eran los incluidos para el estudio (página 114). Por el contrario, en la página 117, en el primer párrafo, después de la descripción de los criterios de exclusión se señala que: "Se incluyeron los artículos que cumplieron los criterios de selección. Dos autores evaluaron de forma independiente los resultados de la búsqueda. Primero examinaron por título y resumen... Cuando un título de un artículo pareció relevante, el resumen fue revisado para su elegibilidad. Si había cualquier duda, se recuperó el texto completo del artículo y fue evaluado para posible inclusión».
A este respecto, no nos parece totalmente claro cuál es el criterio de inclusión: «textos completos publicados", como se indica en la página 114, 0 "abstracts seleccionados que en caso de duda requerirían de la lectura del texto completo del artículo», como a continuación se indica en la página 117. La lectura de este artículo de revisión, efectivamente, confirma que los criterios de inclusión se desvían de lo mencionado en la página 114 hacia lo señalado en la página 117 («lectura del texto completo en caso de que el abstract genere dudas»). Este cambio de criterio se hace obvio cuando en la página 118 se comenta una publicación nuestra y de nuestros colegas ${ }^{2}$ en comparación con el artículo de Egorov, et al. ${ }^{3}$.

Ambas publicaciones tratan de la acción terapéutica del Mexidol, un fármaco de origen ruso para el tratamiento del glaucoma primario de ángulo abierto (POAG, por sus siglas en inglés). Lo primero de todo, nos gustaría llamar su atención por el error cometido cuando es citada nuestra investigación en la revisión de estos autores. El error se basa en el hecho de que la influencia del Mexidol en los cambios del estado anímico de los pacientes con POAG, que se discute en la página 118, no aparece en el artículo que se cita en la revisión sistemática (referencia 24 de la bibliografía). Dichos datos sí aparecen en otra publicación nuestra y de nuestros colegas, en la que realizamos un estudio comparativo de la efectividad de la 3-oxipirodona y los 
derivados del ácido succínico en el tratamiento del $\mathrm{POAG}^{4}$. Creo que usted podría encontrar interesante poder familiarizarse con el texto completo de este artículo.

No tenemos claro por qué cuando los autores discuten este estudio en la página 118 en realidad se refieren a nuestra otra publicación ${ }^{2}$, que se centra exclusivamente en los efectos a nivel ocular del más prometedor derivado de la 3-hidroxipiridina y del ácido succinico (Mexidol), y que no contiene dato alguno relacionado con los estados de ánimo de los pacientes. Además, nos hemos quedado bastante sorprendidos al encontrar en este trabajo de revisión (página 118) la siguiente frase referente a nuestra publicación ${ }^{2}$ "...no había sido incluida en el estudio debido a que no describe el grupo de intervención....". Por el contrario, los autores (Andrea Giaquinta Aranda, Ana Fernández Araque, Rafael Curbelo Rodríguez, Abel Rojo Aragues) sí consideran la inclusión en su estudio del artículo de Egorov, et al. ${ }^{3}$, en el que los resultados del mismo "... coinciden con los datos de Volchegorskii, et al....". Ante esto, sugiero que se comparen las versiones de ambos artículos y sea usted quien, de forma independiente, decida en cuál de ellos el grupo de pacientes tratados es mejor descrito y en cuál los resultados muestran unos datos más concluyentes. Se puede acceder al texto completo original de ambos artículos a través de los enlaces que se muestran en la bibliografía ${ }^{2,3}$.

Creo que merece la pena añadir, además, que, de acuerdo a la tabla 2 de este artículo de revisión, en la cuarta columna de la fila más alta, con respecto al artículo de Egorov, et al. se menciona que «no se declaran los métodos estadísticos empleados", lo que supondría uno de los criterios de exclusión para la revisión sistemática señalados en la página 117.

Opinamos también que esta revisión sistemática presenta errores importantes en el apartado de referencias bibliográficas. En concreto en lo que respecta a las citas 24 y 26, cuyas referencias de paginación son erróneas. Nos gustaría enfatizar que, a los errores de paginación antes referidos, se deben añadir las inexactitudes en la descripción de la bibliografía de relevantes publicaciones procedentes de Enterz-PubMed database, donde solamente se encuentran abstracts traducidos al inglés de importantes publicaciones en lengua rusa. Esto confirmaría un fallo importante a la hora de establecer los criterios de inclusión de acuerdo a lo referido en la página 114 (se analizaron “... publicaciones completas de ensayos clínicos controlados y aleatorizados...").

\section{Bibliografía}

1. Giaquinta Aranda A, Fernández Araque A, Curbelo Rodríguez R, Rojo Aragues A. Glaucoma y antioxidantes: revisión sistemática. Revista Mexicana de Oftalmología. 2017;91:112-21.

2. Volchegorskii IA, Tur EV, Solyannikova OV, et al. The influence of water soluble antioxidant agent (mexidol) on optic nerve and blood flow velocity in ocular and orbital arteries in patients with primary open-angle glaucoma. Vestnik Oftalmologii. 2012;128(4):35-41. Disponible en: https://www.mediasphera.ru/issues/vestnik-oftalmologii/2012/4/downloads/ ru/030042-465x201246. Consultado el 20 de julio de 2017.

3. Egorov EA, Gvetadze AA, Davydova NG. Antioxidant agents in neuroprotection treatment of glaucoma. Vestn Oftalmol. 2013;129(2):67-9. Disponible en: https://www.mediasphera.ru/issues/vestnik-oftalmologii/2013/2/downloads/ru/030042-465X2013214. Consultado el 20 de julio de 2017.

4. Volchegorskii IA, Tur EV, Solyannikova OV, et al. Effectiveness of 3-oxypyridine and succinic acid derivatives in complex treatment of primary-open-angle glaucoma. Eksp Klin Farmakol. 2012;75(7):20-6. Disponible en: http://ekf.folium.ru/index.php/ekf/article/view/184/161. Consultado el 20 de julio de 2017. 